Pacific

Journal of

Mathematics

ANALYTIC CONTINUATION OF CONVEX BODIES AND FUNK'S CHARACTERIZATION OF THE SPHERE

\author{
ERIC L. GRInberg and ERIC Todd Quinto
}




\title{
ANALYTIC CONTINUATION OF CONVEX BODIES AND FUNK'S CHARACTERIZATION OF THE SPHERE
}

\author{
Eric L. Grinberg and Eric Todd Quinto
}

\begin{abstract}
A celebrated theorem of P. Funk, 1916, states that an origincentered star body in $\mathbb{R}^{3}$ is determined by the areas of its central hyperplane cross-sections. In particular, if all these concurrent sections have the same area then the body must be a ball (its boundary is a sphere). It is natural to try to strengthen the theorem by using a smaller class of planes. It is evident that a lower-dimensional class of hyperplanes, e.g., planes passing through an axis, does not suffice, but a proper open subset of planes appears plausible. The class of planes at a small angle relative to an axis has been considered in the literature. We show that this class does not characterize the body. We then show that if a body is known to osculate a ball centered at the origin to infinite order along one hyperplane through the axis, then the proper open class of planes above does determine whether the body is a ball. We generalize our theorem to arbitrary origin centered star bodies and to any open connected collection of planes that fills out $\mathbb{R}^{n}$. We have counterexamples to the theorem for every finite order of osculation. We have similar theorems for the cosine transform and projection areas.
\end{abstract}

\section{Introduction.}

The goal of this paper is to use Radon transforms to answer specific questions about star-shaped and convex sets. Funk showed that a radially symmetric convex body in $\mathbb{R}^{3}$ is determined by areas of its intersections with all planes through the origin. A natural question is whether there are subclasses of planes for which the areas of intersection are sufficient to determine the body. One might think that planes that are within an angle of $\Theta \in(0, \pi / 2)$ of an axis might determine the object, and this claim is in the literature $[\mathbf{T}]$. This is reasonable since the set of all planes near an axis is a full-dimensional set, and every point in the object meets an infinite number of such planes.

In fact, as we show in Proposition 2.2, this does not determine if an object is a ball (i.e., its boundary is a sphere). In fact, Proposition 2.2 shows that even if we assume the object is a ball to any finite order along the hyperplane, it does not have to be a ball everywhere. As a positive result, we prove that 
if an origin-centered star-shaped body is a ball to infinite order along one hyperplane meeting the axis and if its areas are constant on all hyperplanes sufficiently near the axis, then the body is a ball (Theorem 1.2). In fact, our theorem holds for arbitrary origin-centered star bodies that agree to infinite order along a hyperplane through the axis (Theorem 1.3) and for any open connected set of hyperplanes through the origin that fills out $\mathbb{R}^{n}$. We prove similar uniqueness theorems for the cosine transform and projection areas (Theorems 3.1-3.3).

The authors thank Richard Gardner for useful comments and helpful references, in particular to $[\mathbf{S W}]$. This work has also benefited from discussions with Mark Agranovsky, Fulton Gonzalez, Joram Lindenstrauss, and Larry Zalcman. Proposition 2.3 came out of a suggestion of Michael Gage and Frank Morgan, and Maria Moszyńska provided helpful editorial comments. A pleasant conversation and insightful questions by Wolfgang Weil led to a simplification of the proof of Theorem 1.1 and to an improved Proposition 2.1. The authors thank Boris Rubin for specific information about [Mi]. The second author thanks the Humboldt Stiftung for its support and Prof. A.K. Louis and the Fachbereich Mathematik der Universität Saarlandes for their hospitality.

\section{Radon transforms and cross-sections of star bodies.}

A star body is a body in $\mathbb{R}^{n}$ that is star-shaped about the origin; that is, the origin is connected to every point in the body by a segment (which could be just a point) contained in the body. A body $K$ is origin centered if $K=-K$. The sets we consider will be assumed to be compact. We will say two sets are equal when they agree up to a set of measure zero. There is a natural one-to-one correspondence between a star body and its bounding surface, which we will use when convenient.

There is an identification between star shaped bodies in $\mathbb{R}^{n}$ and certain functions on the sphere $S^{n-1}$. If $K$ is a star body in $\mathbb{R}^{n}$ then its radial function $\rho_{K}$ is defined by

$$
\rho_{K}(\omega)=\max \{t \in[0, \infty) \mid t \omega \in K\}
$$

where $\omega \in S^{n-1}$. The number $\rho_{K}(\omega)$ is just the (maximum) distance from the origin to the boundary of $K$ in the direction $\omega$.

For $\omega \in S^{n-1}$ (or for $\omega \in R P^{n-1}$ ) let $P(\omega)$ be the hyperplane through the origin perpendicular to $\omega$ and let $\omega^{\perp}=P(\omega) \cap S^{n-1}$ be the great sphere in $S^{n-1}$ perpendicular to $\omega$.

The Funk-Radon transform for functions $f \in C\left(S^{n-1}\right)$ is defined by

$$
R f(\omega) \equiv \int_{\eta \in \omega^{\perp}} f(\eta) d \eta
$$


Thus $R$ operates by integration on great $(n-2)$ spheres in $S^{n-1}$ with respect to the normalized rotation invariant measure. Call a function $f(\omega)$ even if $f(-\omega)=f(\omega)$. By inspection, $R f$ is always an even function. Minkowski [Mi] proved injectivity of this transform for functions with integrable second derivative. Funk $[\mathbf{F}]$ proved an inversion formula. Both results imply the following uniqueness theorem.

Funk-Minkowski Theorem $([\mathbf{F}, \mathbf{M i}]) . \quad R$ is injective on even functions, $L_{\text {even }}^{2}\left(S^{n-1}\right)$.

It is obvious that $R f$ is zero if $f$ is an odd function. This theorem shows that the null space of $R$ is the set of odd functions. Since $R f$ is always even, we will sometimes view $R$ as an injective transform from $L^{2}\left(R P^{n-1}\right)$ to $L^{2}\left(R P^{n-1}\right)$.

The link between cross-sections of star bodies, $K$, and the transform $R$ is given by the polar coordinate area formula

$$
\text { Area }(K \cap P(\omega))=\frac{1}{n} \int_{\eta \in \omega^{\perp}}\left(\rho_{K}(\eta)\right)^{n-1} d \eta=R\left((1 / n) \rho_{K}^{n-1}\right)(\omega) .
$$

This, in turn, follows from integration in polar coordinates. For background information about star and convex bodies see [G3]. In particular, since star bodies are determined by their radial functions and the Funk-Radon transform is injective on even functions we can make the following assertion. Origin-centered star bodies in $\mathbb{R}^{n}$ are determined by the areas of their central cross-sections.

Funk's original proof of his theorem uses spherical harmonic expansions and is valid on $S^{2}$. After many years it remains the standard proof though others have emerged. See, e.g., [BEGM]. For even functions, Helgason $[\mathbf{H e 1}]$ proved this theorem and inversion formulas for odd dimensional spheres and projective spaces, and Semyanistyi [Se] proved injectivity for spheres of all dimensions using Fourier techniques. Grinberg [Gr] proved injectivity and the inversion formula for spheres of all dimensions (and projective spaces) using spherical harmonics and group invariance.

We need two definitions about asymptotic behavior of functions and sets near a given hypersurface.

Definition 1.1. Let $f$ be a function on a Riemannian manifold and let $S$ be a closed hypersurface. The function $f$ is zero to order $k \in \mathbb{N}$ on $S$ if and only if $f$ is continuous near $S$ and $f(x)=\mathcal{O}\left((\operatorname{dist}(x, S))^{k}\right)$ where $\operatorname{dist}(x, S)$ is the minimum geodesic distance from $x$ to $S$. The function $f$ is zero to infinite order on $S$ if and only if $f$ is zero to order $k$ for all $k \in \mathbb{N}$.

Definition 1.2. Order of osculation among star bodies. Let $K_{1}$ and $K_{2}$ be star bodies in $\mathbb{R}^{n}$, and let $H_{0}$ be a hyperplane through the origin. Assume $K_{1} \cap H_{0}=K_{2} \cap H_{0}$. Let $k \in \mathbb{N} \cup\{\infty\}$. Then, $K_{1}$ and $K_{2}$ osculate to order 
$k$ along $H_{0}$ if and only if the difference of radial functions, $\rho_{K_{1}}-\rho_{K_{2}}$ is zero to order $k$ on $S^{n-1} \cap H_{0}$ according to Definition 1.1.

If the bodies $K_{1}$ and $K_{2}$ have smooth boundaries, then the condition in Definition 1.2 is equivalent to the boundaries of $K_{1}$ and $K_{1}$ being tangent to order $k$ along $K_{1} \cap H_{0}$.

Our first theorem, a support theorem for the Funk-Radon transform, is the key to the subsequent theorems.

Theorem 1.1. Let $A$ be an open connected subset of $S^{n-1}$. Assume $f$ is an even function in $C_{c}\left(S^{n-1}\right)$ with $R f(\omega)=0$ for all $\omega \in A$ and assume, for some $\omega_{0} \in A, f$ is zero to infinite order on $P\left(\omega_{0}\right)$. Then, $f$ is zero on $\cup_{\omega \in A} \omega^{\perp}$.

This theorem can be proven using microlocal techniques [GS] for any generalized Funk-Radon transform (in which the measure $d \eta(1.2)$ is replaced by any nowhere zero real-analytic weight). The transform (1.2) is shown to satisfy a microlocal condition, the Bolker Condition, in [Q1]. Theorem 1.1 is a special case of Theorem 2.2 in [Q3], which is true for all Radon transforms satisfying the Bolker Condition.

However, the proof we give is elementary and it involves the standard projection from a half sphere in $S^{n-1}$ to $\mathbb{R}^{n-1}$. This projection takes great spheres on $S^{n-1}$ into hyperplanes in $\mathbb{R}^{n-1}$ This projection has been used to prove support theorems for the hyperplane and the great-sphere Radon transforms in $[\mathbf{Q 2}]$ and $[\mathbf{B o}]$, and it was noted by Gelfand in the sixties.

Proof of Theorem 1.1. We rotate $S^{n-1} \subset \mathbb{R}^{n}$ so that $\omega_{0}^{\perp}$ is on the $\left(x_{1}, \ldots\right.$, $\left.x_{n-1}\right)$ hyperplane. Then, we consider the projection from the origin through each point in the open upper half sphere $S^{+}$to the $(n-1)$-plane $x_{n}=1$. We identify this plane with $\mathbb{R}^{n-1}$. Under this projection each point $\omega \in$ int $S^{+}$is mapped to the point $x=\frac{1}{\omega_{n}} \omega$ which is identified with its first $n-1$ coordinates, $x^{\prime}=\left(x_{1}, \ldots, x_{n-1}\right)$. The great sphere perpendicular to $\omega$ is mapped to the $n-2$ dimensional hyperplane on $\mathbb{R}^{n-1}$ normal to $\omega^{\prime}=\left(\omega_{1}, \ldots, \omega_{n-1}\right)$ and of distance $\omega_{n} / \sqrt{1-\omega_{n}^{2}}$ from the origin (in the opposite direction to $\left.\omega^{\prime}\right)$.

Let $\mathcal{R}$ be the classical hyperplane transform on $\mathbb{R}^{n-1}$. If $f$ is a continuous even function on $S^{n-1}$ and $\widetilde{f}$ is its projected function on $\mathbb{R}^{n-1}$, and $\omega \in S^{n-1}$, then

$$
\begin{aligned}
& R f(\omega)=\sqrt{1+p^{2}} \mathcal{R}\left(\widetilde{f}(x)\left(1+|x|^{2}\right)^{-(n-1) / 2}\right)(\tau, p) \\
& \text { where } \quad \tilde{f}(x)=f\left(\frac{(x, 1)}{|(x, 1)|}\right), \quad \tau=\frac{\omega^{\prime}}{\left|\omega^{\prime}\right|}, \quad p=\frac{-\omega_{n}}{\sqrt{1-\omega_{n}^{2}}} .
\end{aligned}
$$

The $p$ and $x$ factors in (1.4a) come from the change of coordinates. 
Note that if $f$ is zero to infinite order on $\omega_{0}^{\perp}$, then $\widetilde{f}(x)\left(1+|x|^{2}\right)^{-(n-1) / 2}$ is rapidly decreasing at infinity. Let $\mathcal{A}$ be the set of $n-2$ dimensional hyperplanes in $\mathbb{R}^{n-1}$ that correspond to the set of great spheres parameterized by $A$. As $R f=0$ on $A, \mathcal{R}\left(\tilde{f}\left(1+|x|^{2}\right)^{-(n-1) / 2}\right)$ is zero for all hyperplanes in $\mathcal{A}$. As $A$ is open and $\omega_{0} \in A$, a whole neighborhood of $\omega_{0}$ is in $A$. Since the great sphere $\omega_{0}^{\perp}$ corresponds to the hyperplane at infinity in $\mathbb{R}^{n-1}, \mathcal{A}$ contains all hyperplanes outside of some ball, $B$, in $\mathbb{R}^{n-1}$. Now, we can use Helgason's support theorem for the hyperplane transform $[\mathbf{H e} \mathbf{2}$, Theorem 2.6], which is true for rapidly decreasing functions that are continuous near infinity, to conclude $\widetilde{f}=0$ outside $B$. Finally, we can use the main theorem of $[\mathbf{B Q}]$ (or a generalization of [He2, Lemma 2.11]) to conclude $\tilde{f}$ is zero on $\cup \mathcal{A}$. Therefore, $f$ is zero on $\cup_{(\omega \in A)} \omega^{\perp}$.

Theorem 1.1 has the following consequence for star bodies in $\mathbb{R}^{n}$.

Theorem 1.2. Let $K$ be an origin centered star body in $\mathbb{R}^{n}$ and let $\mathcal{A}$ be an open connected set of hyperplanes through the origin so that $\mathbb{R}^{n}=\cup \mathcal{A}$. If for some $H_{0} \in \mathcal{A}, K$ osculates a ball centered at the origin to infinite order along $H_{0}$ and $K$ has constant cross-sectional areas when sliced by planes in $\mathcal{A}$, then $K$ is that ball.

Note that the collection of planes in $\mathbb{R}^{3}$ within an angle of $\Theta \in(0, \pi / 2)$ of an axis satisfies the hypotheses of Theorem 1.2, so this theorem shows that any set that osculates a ball centered at the origin to infinite order on one 2-plane through the axis and with constant cross-sectional areas on these planes is a ball. Thus, under the additional osculation assumption, the theorem discussed in the introduction $[\mathbf{T}]$ holds.

Proof of Theorem 1.2. Let $A$ be the connected set of directions in $R P^{n-1}$ normal to hyperplanes in $\mathcal{A}, \mathcal{A}=\{P(\omega) \mid \omega \in A\}$. Let $K$ be an origin centered star body. Assume $K$ osculates a ball centered at the origin to infinite order along a plane $H_{0}$, then $\rho_{K}^{n-1}$ is constant to infinite order on $H_{0} \cap S^{n-1}$. If the cross-sectional areas of $K$ are constant on all planes in $\mathcal{A}$, including $H_{0}$, then the Funk transform of $\rho_{K}^{n-1}$ agrees with that of a ball on $A$. By Theorem 1.1, we can conclude the radial function of $K$ is constant on all unit vectors on planes in $\mathcal{A}$. By the assumption that $\mathbb{R}^{n}=\cup \mathcal{A}$, the radial function of $K$ must be constant on $S^{n-1}$ and so $K$ is a ball.

This proof is valid for any pair of origin-centered star bodies that osculate each other to infinite order along a hyperplane, so we obtain the following theorem.

Theorem 1.3. Let $K_{1}$ and $K_{2}$ be origin-centered star bodies in $\mathbb{R}^{n}$ and let $\mathcal{A}$ be an open connected set of hyperplanes through the origin so that $\mathbb{R}^{n}=\cup \mathcal{A}$. If for some $H_{0} \in \mathcal{A}, K_{1}$ and $K_{2}$ osculate each other to infinite 
order along $H_{0}$, and their cross-sectional areas agree when sliced by planes in $\mathcal{A}$, then $K_{1}=K_{2}$.

Simple counterexamples (related to the non-uniqueness of the interior Radon transform on lines) show that the connectedness assumptions in these theorems are necessary.

\section{Counterexamples.}

In this section we explore the hypotheses of the characterization Theorems 1.2 and 1.3, and show through counterexamples that they are needed. First we ask if the 'starter' hypothesis (the condition that the sets agree to infinite order along a hyperplane) can be eliminated entirely. This can be answered using mid-decade developments involving intersection bodies. If $K$ is a star body in $\mathbb{R}^{n}$ then the Funk-Radon transform of its radial function defines another body $I K$, the intersection body of $K$ :

$$
\rho_{I K}(\omega)=R\left(\rho_{K}^{n-1}\right)(\omega),
$$

up to normalizations. H. Busemann proved that if $K$ is a centered convex set, then $I K$ is convex [G3]. Given another star body $L$ it is natural to ask if $L$ is expressible as $I K$ for a star body $K \subset \mathbb{R}^{n}$. In dimension three the answer is affirmative for origin centered bodies [G2]. In higher dimensions the answer is negative $[\mathbf{K o 1}, \mathbf{G 1}, \mathbf{G} \mathbf{2}, \mathbf{Z h}, \mathbf{G Z}]$. These results played a critical role in the solution of the celebrated Busemann-Petty problem. This problem involves the comparison of volumes of two convex bodies by means of comparison of areas of lower-dimensional cross-sections.

In the present context the surjectivity of the intersection body transform in dimension three shows that the 'starter' hypothesis above cannot be entirely eliminated. For the values of central cross-sectional areas of the star body $K$ give the values of the radial function of $I K$. If one could remove the starter hypothesis in Theorem 1.2, then radial functions of star bodies would enjoy a continuation property. Since, in dimension three, every centered convex body is an intersection body, no such continuation can exist. In particular, it is not possible to determine a star body from its central cross-sections by planes with relative angle less than $\Theta$ with respect to an axis since this would imply that an intersection body is determined by its intersection with a central slab. Though intersection bodies form a proper subclass in higher dimensions it is nonetheless possible to show that no suitable continuation property can exist there either.

We now turn to the more delicate question involving the severity of the starter assumption. We will construct a body with constant cross-sections for planes at a small angle relative to an axis which osculates the unit ball to finite order along a plane containing the axis. 
Proposition 2.1. Let $k \in \mathbb{N}$ and let $\Theta \in(0, \pi / 2)$. There is a non-zero function $f \in C_{\text {even }}^{\infty}\left(S^{n-1}\right)$ which vanishes to order $k$ on the great sphere $e_{n}^{\perp}$ (which contains the $x_{1}$-axis) and whose integrals vanish on all great spheres within angle $\Theta$ of the $x_{1}$-axis.

Proof. We use the relation, (1.4), between the great sphere transform and the classical Radon transform on hyperplanes in $\mathbb{R}^{n-1}, \mathcal{R}$, and then a range theorem of Solmon for functions that satisfy a finite number of moment conditions.

Let $H(\tau, p)$ be the hyperplane in $\mathbb{R}^{n-1}$ perpendicular to $\tau \in S^{n-2}$ and $p \geq 0$ units from the origin. Then, under the correspondence (1.4),

$$
H(\tau, p) \quad \text { corresponds to the great sphere } \omega^{\perp} \subset S^{n-1}
$$

$$
\text { where } \omega=\left(\frac{1}{\sqrt{1+p^{2}}} \tau, \frac{-p}{\sqrt{1+p^{2}}}\right) \in S^{n-1} \text {. }
$$

So, the set of great spheres within $\Theta$ radians of the $x_{1}$-axis correspond to $\omega \in S^{n-1}$ with $\left|\omega_{1}\right|<\cos \Theta$. Under (1.4) and (2.1), this set of great spheres corresponds to hyperplanes for $(\tau, p)$ in a subset of the set $\left\{(\tau, p)|| \tau_{1} \mid<\right.$ $a$ or $|p|>b\}$ for some $a>0, b>0$.

We now construct a function $g$ satisfying:

$$
\begin{gathered}
g \in C_{c}^{\infty}\left(S^{n-2} \times \mathbb{R}\right) \\
g(\tau, p)=g(-\tau, p)=g(-\tau,-p)
\end{gathered}
$$

$g$ is supported in $C=\left\{(\tau, p)|| \tau_{1} \mid>a\right.$ and $\left.0<|p|<b\right\}$

$$
\int_{p=-\infty}^{\infty} g(\tau, p) p^{m} d p=0 \quad \forall \tau \in S^{n-2}, m=0,1, \ldots, k .
$$

Let $g_{1}(\tau)$ be a smooth even function that is supported in $\left\{\tau \in S^{n-2}|| \tau_{1} \mid>\right.$ $a\}$. It is easy to construct a function $g_{2}(p) \in C_{c}^{\infty}((0, b))$ that satisfies $\int_{p=-\infty}^{\infty} g_{2}(p) p^{m} d p=0, \forall m=0, \ldots, k$. For example, one can just take an appropriate linear combination of $k+2$ translates of a small bump function supported in $(0, b /(k+4))$. Now, extend $g_{2}$ to $\mathbb{R}$ to be even. Let $g(\tau, p)=g_{1}(\tau) g_{2}(p)$ and then $g$ satisfies $(2.2 \mathrm{a})-(2.2 \mathrm{~d})$.

Now, we use Theorem 7.7 , p. 376 of [So] to conclude that $g=\mathcal{R} h$ for some $h \in C^{\infty}\left(\mathbb{R}^{n-1}\right)$. Furthermore, because $g$ satisfies the first $k$ moment conditions, $(2.2 \mathrm{~d})$,

$$
h=\mathcal{O}\left(|x|^{-n-k}\right) \text { at infinity. }
$$

Next, we let $f: S^{n-1} \rightarrow \mathbb{R}$ be defined by

$$
f(\omega)=h\left(\frac{\omega^{\prime}}{\omega_{n}}\right)\left|\omega_{n}\right|^{-(n-1)}
$$


Then $f$ is zero to order $k$ on $e_{n}^{\perp}$ by the relation of $f$ to $h,(2.4)$, and the growth condition on $h,(2.3)$ (and since $|x|=1 /\left|\omega_{n}\right|$ ). Also, $f$ is continuous on $S^{n-1}$ and $f$ is smooth away from $e_{n}^{\perp}$.

Finally, we look back at (1.4) to see that

$$
R f(\omega)=\left(1-\omega_{n}^{2}\right)^{-1 / 2} g\left(\omega^{\prime} /\left|\omega^{\prime}\right|,-\omega_{n} / \sqrt{1-\omega_{n}^{2}}\right) .
$$

Because of (1.4) and the definition of $g, R f(\omega)=0$ for $\left|\omega_{1}\right|<\cos \Theta$. By $(2.2 \mathrm{~b})-(2.2 \mathrm{c}) g(\tau, p)$ is zero near $p=0\left(\omega_{n}=0\right)$ and $g$ is zero near $p=\infty$ $\left(\omega_{n}=1\right)$ and $g$ is even. Therefore, $R f$ is smooth and even on $S^{n-1}$. Since $R$ is bijective on $C_{\text {even }}^{\infty}\left(S^{n-1}\right)$ (and injective on $\left.C\left(S^{n-1}\right)\right), f$ is smooth. This finishes the construction.

Let $B_{n}$ be the unit ball in $\mathbb{R}^{n}$. Our next proposition is an application Proposition 2.1 to convex sets.

Proposition 2.2. Let $k \in \mathbb{N}$ and let $\Theta \in(0, \pi / 2)$. Let $H_{0}$ be the hyperplane in $\mathbb{R}^{n} x_{n}=0$. There is a strictly convex origin-centered set, $K$, with smooth boundary that is not a ball but that osculates the unit ball, $B_{n}$, to order $k$ along $H_{0}$ and which has cross-sectional areas of $\pi$ on all hyperplanes through the origin and within $\Theta$ radians of the $x_{1}$-axis.

Proof. Let $f$ be an even non-zero function satisfying the conclusions of Proposition 2.1. Let $\epsilon_{0}>0$ be so small that $1+\epsilon_{0} f>0$. By the choice of $\epsilon_{0}$, for any $\epsilon \in\left(0, \epsilon_{0}\right), \rho_{\epsilon}(\omega)=\sqrt[n-1]{1+\epsilon f(\omega)}$ is the radial function of an origin-centered set, $K_{\epsilon}$ with smooth boundary. Since the unit sphere has strictly positive curvature, for sufficiently small $\epsilon \in\left(0, \epsilon_{0}\right), K_{\epsilon}$ will be strictly convex; see the end of the proof of Theorem 3.1 of [G1] and also $[\mathbf{O}]$.

These counterexamples give the following strong non-uniqueness results.

Proposition 2.3. Let $A$ be any set in $S^{n-1}$ that omits an even open subset of $S^{n-1}$. Then, $f \in C_{\text {even }}^{\infty}\left(S^{n-1}\right)$ is not determined by data $R f(\omega)$ for $\omega \in$ $A$. Furthermore, origin centered convex bodies are not determined by crosssectional areas on subspaces perpendicular to vectors in $A$.

Proof. Any set $A$ that omits an even open subset of $S^{n-1}$ can be rotated so that the omitted subset contains the $x_{1}$-axis. Proposition 2.1 provides the non-uniqueness result for the Funk-Radon transform. Proposition 2.2 provides the non-uniqueness result for cross-sectional areas.

\section{Projections of convex bodies and the cosine transform.}

Now, we explain how the projection areas of a convex body give the Cosine transform of the surface area measure of the body. We review the relation between the Cosine and Funk-Radon transform and note that the two are related by an invertible real analytic elliptic differential operator and hence 
have the same microlocal support properties. We can then state analogs of the cross-section theorems above for projection areas ("shadows").

We would like to formulate analogs of the results of Section 1 for projections or shadows instead of cross-sections. If $K$ is a star body in $\mathbb{R}^{n}$ let $\left.K\right|_{P(\omega)}$ denote its orthogonal projection into the hyperplane, $P(\omega)$, through the origin which has normal vector $\omega$. The quantity $\operatorname{Area}\left(\left.K\right|_{P(\omega)}\right)$ is called the brightness of $K$ in the direction $\omega$. Elementary examples show that we can only hope to recover the convex hull of $K$ from its brightness function and, in keeping with tradition, we restrict the discussion to convex bodies $K$. There is a partial duality relating cross-sections of star bodies and projections of convex bodies.

With each smooth convex body $K$ we can associate a measure on $S^{n-1}$. To define it, we first define the Gauss map to be the map $g_{K}:$ bd $K \rightarrow S^{n-1}$ taking $x \in \operatorname{bd} K$ to the outer unit normal to bd $K$ at $x$. If $\mathrm{bd} K$ is $C^{1}$ and strictly convex, then $g_{K}$ is continuous and bijective [G3, p. 24-5]. If bd $K$ is $C^{2}$ and strictly positively curved everywhere then $g_{K}$ is a $C^{1}$ function with $C^{1}$ inverse (the inverse is $C^{1}$ because strict positive curvature implies the derivative of $g_{K}$ has only positive eigenvalues). If bd $K$ is $C^{\infty}$ and strictly positively curved everywhere then $g_{K}$ is a $C^{\infty}$ function with $C^{\infty}$ inverse.

This allows us to define a measure, $S_{K}$, on $S^{n-1}$, the infinitesimal surface area measure of $K$. Let $E \subset S^{n-1}$ be measurable and let $\lambda_{n-1}$ be the surface area measure on bd $K$. Then the measure is defined by

$$
S_{K}(E)=\lambda_{n-1}\left(g_{K}^{-1}(E)\right) .
$$

In integrals, we will denote this measure by $d S_{K}$.

Note that the infinitesimal surface area measure of the unit ball is the standard measure on the sphere because the Gauss map is the identity. If bd $K$ is strictly positively curved and $C^{2}$, then calculation (3.7) below shows that $d S_{K}$ is absolutely continuous with respect to Lebesgue measure on the sphere. However, the infinitesimal surface measure can be defined even if the Gauss map is not well-defined (e.g., if $K$ is a polytope). See [G3] for details.

This measure is important because the projection area

$$
\operatorname{Area}\left(\left.K\right|_{P(\omega)}\right)=\int_{S^{n-1}}|\omega \cdot \eta| d S_{K}(\eta)
$$

where $\omega \cdot \eta$ is the Euclidean inner product of these vectors.

If $K$ is origin-centered then $K$ is determined by its infinitesimal surface area measure. This follows from the Alexandrov projection and uniqueness theorems [G3, §3.3]. If $K$ is smoothly bounded and positively curved throughout, then $d S_{K}$ is absolutely continuous with respect to Lebesgue measure on $S^{n-1}$ and its Radon-Nikodym derivative is the $(n-1)^{\text {st }}$ elementary symmetric function of the principal radii of curvature of $K[\mathbf{S 2}, \S 2.5$, 
4.2]. The projection areas of the convex body $K$ define a centered body $\Pi(K)$ called the projection body of $K$, by duality with intersection bodies above. We refer to [G3] for general background on these matters.

Equation (3.1) suggests an integral operator called the Cosine transform. Its definition is:

$$
\begin{gathered}
T: C\left(S^{n-1}\right) \rightarrow C_{\text {even }}\left(S^{n-1}\right) \\
T f(\omega) \equiv \int_{S^{n-1}}|\omega \cdot \eta| f(\eta) d \eta,
\end{gathered}
$$

where $d \eta$ is the rotation invariant normalized measure on the sphere. The cosine transform is a special Blaschke-Levy Representation, and many authors, including Alexandrov, Gardner, Goodey, Groemer, Koldobsky, Rubin, Schneider, and Weil have proven important properties including inversion formulas [Ko2] and inversion formulas using wavelets [Ru1] and relations to other transforms $[\mathbf{R u 2}]$. See [G3, Ko2] for more information.

There is an identity linking the Funk-Radon and Cosine transforms $[\mathbf{G W}]$ :

$$
(\Delta+2(n-1)) T f(\omega)=2 R f(\omega), \text { for } \omega \in S^{n-1} .
$$

Here $\Delta$ denotes the spherical Laplacian. Thus $R$ and $T$ are related by an invertible real-analytic-elliptic operator and so they have the same microlocal transformation properties. This means, in particular, that $R f$ is smooth or real-analytic whenever $T f$ is. More precisely, $T f$ and $R f$ have the same wavefront set in both the smooth $[\mathbf{H o ̈}]$ and real-analytic $[\mathbf{T r}]$ categories. The relation (3.4) gives us analogous theorems to Theorem 1.2 and 1.3 for the Cosine Transform. For $\omega \in S^{n-1}$, recall that $P(\omega)$ is the hyperplane through the origin in $\mathbb{R}^{n}$ perpendicular to $\omega$ and $\omega^{\perp}=P(\omega) \cap S^{n-1}$.

Theorem 3.1. Let $K_{1}$ and $K_{2}$ be origin centered convex bodies in $\mathbb{R}^{n}$ with $C^{2}$ boundaries that are strictly positively curved. Let $A$ be an open connected set in $S^{n-1}$ such that $\mathbb{R}^{n}=\cup_{(\omega \in A)} P(\omega)$. Assume that for some $\eta_{0} \in A$, the infinitesimal surface area measures of $K_{1}$ and $K_{2}$ agree to infinite order ${ }^{1}$ along the equator $\eta_{0}^{\perp}$, and assume the projection areas (3.2) of $K_{1}$ and $K_{2}$ agree for $\omega \in A$. Then $K_{1}=K_{2}$.

The assumptions about $K_{j}$ in the theorem ensure that the Radon-Nikodym derivatives of the infinitesimal surface area measures with respect to Lebesgue measure exist.

Proof. For $j=1,2$, because $K_{j}$ is $C^{2}$ and strictly positively curved, its infinitesimal surface measure is absolutely continuous with respect to Lebesgue measure on the sphere (see (3.7)). Let $f_{j}$ be its Radon-Nikodym derivative with respect to Lebesgue measure; then $f_{j}$ is a continuous function [G3]. The identities (3.2) and (3.4) can be used to show that the Funk-Radon

\footnotetext{
${ }^{1}$ That is, the Radon-Nikodym derivatives of $d S_{K_{1}}$ and $d S_{K_{2}}$ with respect to Lebesgue measure on the sphere are functions that agree to infinite order according to Definition 1.1.
} 
transforms of the functions $f_{1}$ and $f_{2}$ are identical on $A$. Since $f_{1}$ and $f_{2}$ agree to infinite order on the great sphere $\eta_{0}^{\perp}$ and $\eta_{0} \in A$, Theorem 1.1 can be used to finish the proof.

Our next theorem follows from Theorem 3.1, and it has a more geometric starter condition.

Theorem 3.2. Let $K$ be an origin centered convex body in $\mathbb{R}^{n}$ with a $C^{2}$ boundary that is strictly positively curved. Let $A$ be an open connected set in $S^{n-1}$ such that $\mathbb{R}^{n}=\cup_{(\omega \in A)} P(\omega)$. Assume that for some $\eta_{0} \in A, K$ osculates a disk, $D$, centered at the origin to infinite order along $P\left(\eta_{0}\right)$ and assume the projection areas (3.2) of $K$ agree on $A$ with those of $D$. Then $K=D$.

Schneider $[\mathbf{S} 1]$ has proven important related theorems. Let $K_{1}$ be a centered, convex polytope. Assume that $K_{2}$ is a centered convex body and the projection areas of $K_{1}$ and $K_{2}$ agree for all $\eta$ in an open set $A$ of arbitrarily small measure (and containing the normal vectors to each face of $K_{1}$ ). He proves that $K_{1}$ and $K_{2}$ are the same. His starter assumption is that $K_{1}$ is a polytope, but he does not assume bd $K_{1}$ and bd $K_{2}$ agree anywhere, and his set $A$ is different from ours.

Schneider and Weil prove a related theorem in $[\mathbf{S W}]$ for odd dimensions. Their starter assumption is that each body has at least one vertex and both sets share a supporting plane, $\mathcal{P}$, that touches a vertex of each body. They assume the projection areas of $K_{1}$ and $K_{2}$ are the same for normal vectors in an equatorial belt perpendicular to the normal vectors to $\mathcal{P}$. Then, they conclude $K_{1}=K_{2}$. They also show that either if $n$ is even or if only one of the sets has a vertex supporting plane, then the theorem is false. This very intriguing theorem has a different flavor from ours since convex sets with vertices do not have smooth boundaries, so our theorem does not apply. Also, their set $A$ is different from ours since it does not include the vector for which starter information is given (the normal to $\mathcal{P}$ ).

Proof. We can assume the disk $D$ is the unit disk in $\mathbb{R}^{n}$. We show that the infinitesimal surface area measure of $K$ agrees on $\eta_{0}^{\perp}$ to infinite order with that of $D$. Then, we use Theorem 3.1 to conclude that $K=D$.

To compare $d S_{K}$ to $d \eta=d S_{D}$, we use (3.2) to write the surface area on bd $K, \lambda_{n-1}$, as a measure on $S^{n-1}$ using the radial map $\rho_{K}: S^{n-1} \rightarrow \mathbb{R}$. Recall that the Gauss map $g_{K}:$ bd $K \rightarrow S^{n-1}$ takes points on bd $K$ to their unit outer normals. Let $h: S^{n-1} \rightarrow$ bd $K$ be defined by

$$
h(\omega)=\rho_{K}(\omega) \omega .
$$

A straightforward exercise shows for $F \subset$ bd $K$ that

$$
\lambda_{n-1}(F)=\int_{h^{-1}(F)} \frac{\rho_{K}^{n-1}(\omega)}{\left|\omega \cdot g_{K}(h(\omega))\right|} d \omega .
$$


Another exercise using a change of variables (where $J$ is the Jacobian determinant) shows that if $E \subset S^{n-1}$ measurable, then

$$
\begin{aligned}
S_{K}(E) & =\int_{\omega \in\left(g_{K} \circ h\right)^{-1}(E)} \frac{\rho_{K}^{n-1}(\omega)}{\left|\omega \cdot g_{K}(h(\omega))\right|} d \omega \\
& =\left.\int_{\eta \in E}\left[\frac{\rho_{K}^{n-1}(\omega)}{\left|\omega \cdot g_{K}(h(\omega))\right|} \frac{1}{\left|J\left(g_{K} \circ h\right)(\omega)\right|}\right]\right|_{\omega=\left(g_{K} \circ h\right)^{-1}(\eta)} d \eta .
\end{aligned}
$$

It should be pointed out that $J\left(g_{K} \circ h\right)$ is non-zero since $K$ is strictly convex and $C^{2}$. Since $K$ osculates the unit disk along $P\left(\eta_{0}\right)$ to infinite order, this implies $\rho_{K}=1$ to infinite order on the great sphere $\eta_{0}^{\perp}$ and that $g_{K} \circ h$ is the identity map to infinite order on $\eta_{0}^{\perp}$. This implies that the expression in brackets in (3.7) is equal to one to infinite order on $\eta_{0}^{\perp}$. But, this expression is just the Radon-Nikodym derivative of $S_{K}$. Therefore, the hypotheses of Theorem 3.1 hold and we can use that to prove our theorem.

A similar result to Theorem 3.2 can be stated for arbitrary strictly convex sets $K_{1}$ and $K_{2}$ that agree to infinite order on a set that becomes a great sphere under the Gauss map.

Theorem 3.3. Let $K_{j}$ be an origin centered convex body in $\mathbb{R}^{n}$ with a $C^{\infty}$ boundary that is strictly positively curved for $j=1,2$. Let $A$ be an open connected set in $S^{n-1}$ so that $\mathbb{R}^{n}=\cup_{(\omega \in A)} P(\omega)$. Let $\eta_{0} \in A$, and assume $K_{1}$ osculates $K_{2}$ to infinite order along the set $H=g_{K_{1}}^{-1}\left(\eta_{0}^{\perp}\right)$. Assume the projections (3.2) of $K_{1}$ and $K_{2}$ agree on $A$. Then $K_{1}=K_{2}$.

Since $K_{j}$ is strictly convex with $C^{\infty}$ boundary, the set $H=g_{K_{1}}^{-1}\left(\eta_{0}^{\perp}\right)$ is smooth. The osculation condition in Theorem 3.3 is defined in terms of the radial functions of $K_{1}$ and $K_{2}$ in a similar way as in Definitions 1.1 and 1.2.

The proof is similar to the proof of Theorem 3.2. One observes that, if $K_{1}$ osculates $K_{2}$ to infinite order along $H$, then the radial maps $\rho_{K_{j}}$ agree to infinite order on $h_{j}^{-1}\left(H \cap \operatorname{bd} K_{j}\right)=H \cap S^{n-1}$ (where $h_{j}: S^{n-1} \rightarrow$ bd $K_{j}$ is the map defined by (3.5)) and the Gauss maps $g_{K_{j}}$ agree to infinite order on $H \cap \mathrm{bd} K_{j}$. Then, one can use (3.7) to observe that the infinitesimal surface area measures agree to infinite order on $\eta_{0}^{\perp}$. Finally, one uses Theorem 3.1.

\section{References}

[BEGM] T.N. Bailey, M.G. Eastwood, A.R. Grover and L.J. Mason, The Funk Transform as a Penrose Transform, Mathematical Proceedings of the Cambridge Philosophical Soc., 125 (1999), 67-81, MR 99i:32037, Zbl 958.44002.

[Bo] J. Boman, Helgason's support theorem for Radon transforms - a new proof and a generalization, in 'Mathematical Methods in Tomography,' Proceedings, Oberwolfach, 1990, Lecture Notes in Mathematics, vol. 1497, Springer Verlag, Berlin, New York, 1991, 1-5, CMP 1178 765, Zbl 772.44003. 
[BQ] J. Boman and E.T. Quinto, Support theorems for real analytic Radon transforms, Duke Math. J., 55 (1987), 943-948, MR 89m:44004, Zbl 645.44001.

[F] P. Funk, Über eine geometrische anwendung der abelschen integralgleichung, Math. Ann., 77 (1916), 129-135.

[G1] R.J. Gardner, Intersection bodies and the Busemann-Petty problem, Trans. Amer. Math. Soc., 342(1) (1994), 435-444, Zbl 801.52005.

[G2] A positive answer to the Busemann-Petty problem in three-dimensions, Annals Math., 140 (1994), 435-447, MR 94e:52008, Zbl 826.52010.

[G3] , Geometric Tomography, Camb. U. Press, New York, 1995, MR 96j:52006, Zbl 864.52001.

[GW] P. Goodey and W. Weil, Centrally symmetric convex bodies and the spherical Radon transform, J. Diff. Geom., 35 (1992), 675-688, MR 93g:44005, Zbl 780.52004.

[Gr] E.L. Grinberg, Spherical harmonics and integral geometry on projective spaces, Trans. Amer. Math. Soc., 279 (1983), 187-203, MR 84m:53071, Zbl 518.43006.

[GZ] E.L. Grinberg and G. Zhang, Convolutions, transforms and convex bodies, Proc. London Math. Soc., (1999), 77-115, MR 99m:52009.

[GS] V. Guillemin and S. Sternberg, Geometric Asymptotics, Amer. Math. Soc., Providence, RI, 1977, MR 58 \#24404, Zbl 364.53011.

[He1] S. Helgason, Differential operators on homogeneous spaces, Acta Math., 102 (1959), 239-299, MR 22 \#8457, Zbl 146.43601.

[He2] - The Radon Transform, Second Edition, Birkhäuser, Boston, 1999, MR 2000m:44003, Zbl 932.43011.

[Hö] L. Hörmander, The Analysis of Linear Partial Differential Operators I, Springer Verlag, New York, 1983, MR 85g:35002a, Zbl 521.35001.

[Ko1] A. Koldobsky, Intersection bodies and the Busemann-Petty problem, C.R. Acad. Sci. Paris, Ser. Math., 325 (1997), 1181-1186, MR 98k:52007, Zbl 898.52001.

[Ko2] _ Inverse formula for the Blaschke-Levy representation, Houston J. Math., 23 (1997), 95-107, MR 2000b:42005.

[L] E. Lutwak, Inequalities for Hadwiger's harmonic quermassintegrals, Math. Ann., 280 (1998), 165-175, MR 89h:52005.

[Mi] H. Minkowski, On bodies of constant width (in Russian), Mat. Sbornik, 25 (1904), 505-508; German translation: Gesammelte Abhandlungen, 2, Teubner, Leipzig, (1911), 277-279.

[O] V. Oliker, Hypersurfaces in $\mathbb{R}^{n+1}$ with prescribed Gaussian curvature and related equations of Monge-Ampére type, Comm. Partial Diff. Eq., 9 (1984), 807-838, MR 85h:53047, Zbl 559.58031.

[Q1] E.T. Quinto, Topological restrictions on double fibrations and Radon transforms, Proc. Amer. Math. Soc., 81 (1981), 570-574, MR 82g:58084, Zbl 424.58010.

[Q2] - The invertibility of rotation invariant Radon transforms, J. Math. Anal. Appl., 91 (1983), 510-522, MR 84j:44007a, Zbl 517.44009; Erratum, J. Math. Anal. Appl., 94 (1983), 602-603, MR 84j:44007b, Zbl 517.44010.

[Q3] _ Radon transforms satisfying the Bolker assumption, in Proceedings of conference 'Seventy-five Years of Radon Transforms', International Press Co. Ltd., Hong Kong, 1994, 263-270, MR 95k:58160, Zbl 823.44008. 
[Ru1] B. Rubin, Fractional integrals and wavelet transforms associated with BlaschkeLevy representations on the sphere, Israel Journal of Math., 114 (1999), 1-27, MR 2001b:42054.

[Ru2] Inversion and characterization of the hemispherical transform, Journal D'Analyse Math., 77 (1999), 105-128, CMP 1753484.

[S1] R. Schneider, On the projections of a convex polytope, Pacific J. Math., 32 (1970), 799-803, MR 42 \#2363, Zbl 187.44201.

[S2] Convex Bodies: The Brunn-Minkowski Theory, Camb. Univ. Press, Cambridge, 1993, MR 94d:52007, Zbl 798.52001.

[SW] R. Schneider and W. Weil, Über die bestimmung eines konvexen körpers durch die inhalte seiner projektionen, Math Z., 116 (1970), 338-348, MR 44 \#922, Zbl 197.18601.

[Se] V.I. Semyanistyi, Homogeneous functions and some problems of integral geometry in spaces of constant curvature, Sov. Math. Dokl., 2 (1961), 59-62, MR 24 \#A2842, Zbl 100.36602.

[So] D. Solmon, Asymptotic formulas for the dual Radon transform and applications, Math. Z., 195 (1987), 321-343, MR 88i:44006, Zbl 598.44001.

[T] L. Tamássy, A characteristic property of the sphere, Pacific J. Math., 29(2) (1969), 439-446, MR 39 \#7543, Zbl 181.23702.

[Tr] F. Treves, Introduction to Pseudodifferential and Fourier Integral Operators I, II, Plenum Press, New York, 1980, MR 82i:35173, MR 82i:58068, Zbl 453.47027.

[Zh] G. Zhang, Sections of convex bodies, Amer. J. Math., 118 (1996), 319-340, MR 97f:52015, Zbl 854.52004.

Received February 16, 1999. The first author was partially supported by NSF grant 9971828, and the second author was partially supported by NSF grants 9622947 and 9877155 .

Department of Mathematics

Temple University

Philadelphia, PA 19122

E-mail address: grinberg@math.temple.edu

Department of Mathematics

TUFTS UNIVERSITY

MEdFord, MA 02155

E-mail address: equinto@tufts.edu 